\title{
Identification of differentially expressed proteins in the locoregional recurrent esophageal squamous cell carcinoma by quantitative proteomics
}

\author{
Wei-Wei Yu ${ }^{1}$, Xiao-Long Fu ${ }^{2}$, Xu-Wei Cai ${ }^{2}$, Meng-Hong Sun ${ }^{3}$, Yan-Mei Guo ${ }^{4}$ \\ ${ }^{1}$ Department of Radiation Oncology, Affiliated Hospital of Nantong University, Nantong, China; ${ }^{2}$ Department of Radiation Oncology, Shanghai \\ Chest Hospital, Shanghai Jiao Tong University, Shanghai, China; ${ }^{3}$ Department of Pathology, Fudan University Shanghai Cancer Center, Shanghai, \\ China; ${ }^{4}$ Department of Gastroenterology, The Tenth People's Hospital of Tongji University, Shanghai, China \\ Contributions: (I) Conception and design: YM Guo, XL Fu; (II) Administrative support: XL Fu; (III) Provision of study materials or patients: MH \\ Sun, WW Yu; (IV) Collection and assembly of data: WW Yu; (V) Data analysis and interpretation: YM Guo, XW Cai; (VI) Manuscript writing: All \\ authors; (VII) Final approval of manuscript: All authors. \\ Correspondence to: Yan-Mei Guo. Department of Gastroenterology, The Tenth People's Hospital of Tongji University, 301 Middle Yan Chang Rd., \\ Shanghai 200072, China. Email: guoyanmei100@126.com.
}

\begin{abstract}
Background: This study aimed to identify potential biomarkers associated with locoregional recurrence in patients with esophageal squamous cell carcinoma (ESCC) after radical resection.

Methods: We performed a quantitative proteomics analysis using isobaric tags for relative and absolute quantification (iTRAQ) with reversed-phase liquid chromatography-mass spectrometry (RPLC-MS) to identify differential expression proteins (DEPs) between a locoregional recurrence group and good prognosis group of ESCC after radical esophagectomy. The bioinformatics analysis was performed with ingenuity pathway analysis software (IPA) and Gene Ontology (GO) database using the software of MAS 3.0. KaplanMeier (KM) Plotter Online Tool (http://www.kmplot.com) was used to evaluate the relationship between the differential expression of proteins and survival in patients with ESCC.

Results: More than 400 proteins were quantitated of which 27 proteins had upregulated expression and 55 proteins had downregulated expression in the locoregional recurrence group compared to the good prognosis group. These 82 DEPs were associated with biological procession of cancer development including cellular movement, cellular assembly and organization, cellular function and maintenance, cellular growth and proliferation, cell death and survival, DNA replication recombination and repair, and so on. Of these DEPs, SPTAN1 and AGT proteins were identified to be associated with RFS in ESCC. SPTAN1 was positively associated with RFS and AGT was negatively associated with RFS. Expression of SPTAN1 tended to have favorable OS while expression of AGT tended to have poor OS.

Conclusions: Our results demonstrated that quantitative proteomics is an effective discovery tool to identify biomarkers for prognosis prediction in ESCC. However, it needs more studies with large populations of ESCC to validate these potential biomarkers.
\end{abstract}

Keywords: Biomarkers; esophageal carcinoma; quantitative proteomics; recurrence

Submitted Mar 29, 2021. Accepted for publication Jun 15, 2021.

doi: 10.21037/jgo-21-278

View this article at: https://dx.doi.org/10.21037/jgo-21-278

\section{Introduction}

Despite the development of surgical techniques, the 5-year overall survival (OS) of patients with esophageal squamous cell carcinoma (ESCC) has remained dismal (1-2). The poor prognosis is generally related to locoregional recurrence (3). To date, several studies have shown that surgery combined 
with adjuvant or neoadjuvant chemoradiotherapy has significantly improved prognosis (4-7). However, these multidisciplinary treatment modalities can cause serious side effects and benefit only a limited number of patients in terms of local control and OS. In addition, the adjuvant treatment would be unnecessary for patients who could be cured by surgery alone. Thus, accurate risk stratification is essential to avoid potential morbidity due to overtreatment or to prevent further development of disease. Nevertheless, until now, efforts to identify appropriate prognostic factors for high risk population screening in ESCC have proven to be primarily unsuccessful $(8,9)$.

Proteomics, concerning comprehensive protein profile changes caused by multigene alterations, is currently considered the most powerful tool for thorough assessment of protein expression and screening of novel cancer-specific biomarkers $(10,11)$. Tissue based proteomics analyses directly relate protein biomarkers to disease and have been applied in the study of various tumors $(12,13)$. Several previous studies have explored the differences in protein expression between esophageal squamous cell carcinoma and paracancerous tissues through proteomics (14). Zhang et al. (15) have analyzed ESCC tissues and adjacent normal tissues by two-dimensional electrophoresis and matrixassisted laser desorption/ionization-time-of-flight mass spectrometry (MALDI-TOF MS) analysis. The results displayed that a total of 104 protein spots with different expression levels were found on $2 \mathrm{DE}$, and 47 proteins were eventually identified. Among these identified proteins, 33 proteins were over-expressed and 14 proteins were downexpressed in ESCC.

Recently, Isobaric tags for relative and absolute quantification (iTRAQ) method used as a multiplexed quantitative proteomic labeling strategy has been widely applied to global analysis in diverse cancers. iTRAQ method is a highly sensitive proteomic platform with high proteome coverage and labeling efficiency, and has no side effects on the biochemical properties of the labeled proteins or peptides (16). In this study, we performed a tissue-based quantitative proteomic analysis to search differential expression proteins (DEPs) involved in locoregional recurrence in ESCC after curative resection using iTRAQ method coupled with strong cation exchange (SCX)-reversed phase liquid chromatography-tandem mass spectrometry (RPLC-MS/MS). The aim of this study was to screen DEPs relating to locoregional recurrence. Therefore, we mainly compared protein expression profiles between ESCC with locoregional recurrence and ESCC without locoregional recurrence after radical surgery. We presented the following article in accordance with the REAMRK reporting checklist (available at https://dx.doi. org/10.21037/jgo-21-278).

\section{Methods}

\section{Sample collection}

All fresh tissues were obtained from ESCC patients who underwent radical surgery at Fudan University Cancer Center from October 2007 to September 2009. The main inclusion criteria for these patients were as follows: (I) confirmed ESCC by histopathology, (II) classified according to the sixth edition of tumor, nodes, metastasis-Union for International Cancer Control/American Joint Committee on Cancer (TNM-UICC/AJCC) staging system, (III) only 1 primary tumor, (IV) not receiving neoadjuvant chemotherapy and/or radiotherapy, $(\mathrm{V})$ underwent complete 3 -field lymphadenectomy (3FLND) with $\geq 15$ total lymph nodes removed; and (VI) confirmed R0 resection by histopathology. As the purpose of this study was to screen DEPs relating to locoregional recurrence, patients who had accepted adjuvant radiotherapy and/or chemoradiotherapy were excluded to eliminate the effects of local treatment on locoregional control. All procedures performed in this study involving human participants were in accordance with the Declaration of Helsinki (as revised in 2013). The study was approved by the appropriate institutional review board of Fudan University Cancer Center and informed consent was taken from all the patients.

All participants were followed up for at least 3 years after surgery. Participants who survived for more than 3 years without evidence of disease recurrence were classified in the good prognosis group $(n=7)$; those who relapsed in the local primary tumor site and/or regional lymph nodes within 2 years after surgery were categorized as the locoregional recurrence group $(n=8)$; the proteomic profiles of the 2 groups were compared. Samples of ESCC tissues from the 2 groups were obtained during operation, snap frozen at $-80^{\circ} \mathrm{C}$ and stored until use.

\section{Protein extraction}

The tissue samples (50 $\mathrm{mg}$ for each sample) from the same group were mixed together for proteomic analysis. Fresh frozen ESCC tissue samples were cut into small pieces, ground in liquid nitrogen, and dissolved in lysis buffer at 
the ratio of $1 \mathrm{mg}$ of tissue per $5 \mu \mathrm{L}$ of lysis buffer [ $7 \mathrm{M}$ urea, $2 \mathrm{M}$ sulfocarbamide, $0.1 \mathrm{mmol} / \mathrm{L}$ phenylmethylsulfonyl fluoride, $65 \mathrm{mM}$ dithiothreitol (DTT)]. The protein mixtures were swirled vigorously for $30 \mathrm{~min}$ with frequent breaks of ice-incubation, followed by centrifuging at $14,000 \mathrm{rpm}$ for $30 \mathrm{~min}$ at $4{ }^{\circ} \mathrm{C}$. The supernatants were stored in aliquots at $-80^{\circ} \mathrm{C}$. The protein concentrations were measured using a Bradford Protein Assay (Bio-Rad Laboratories, Inc., Hercules, CA, USA) (17).

\section{Protein digestion and $i T R A Q$ reagent labeling}

The iTRAQ labeling was performed according to the kit protocol (Applied Biosystems, Forster City, CA, USA). A total of $100 \mu \mathrm{g}$ proteins of each group was added with 4 times the volume of cold $\left(-20^{\circ} \mathrm{C}\right)$ acetone, and the mixture was precipitated at $-20^{\circ} \mathrm{C}$ for 1 hour. Then, the mixture was centrifuged at $12,000 \mathrm{rpm}$ at $4{ }^{\circ} \mathrm{C}$ for $15 \mathrm{~min}$ and the supernatant was removed. The protein pellets were then dissolved in the buffer solution $(20 \mu \mathrm{L})$ and $1 \%$ sodium dodecyl sulfate (SDS) $(1 \mu \mathrm{L})$, reduced in $2 \mu \mathrm{L}$ of reducing reagent for 1 hour at $60{ }^{\circ} \mathrm{C}$, and blocked with cysteines in $1 \mu \mathrm{L}$ of cysteine blocking reagent for $10 \mathrm{~min}$. Each sample was digested with sequencing-grade modified trypsin (Sigma, St. Louis, MO, USA) with a protein-toenzyme ratio of $20: 1$ at $37^{\circ} \mathrm{C}$ overnight, then labeled with iTRAQ reagents for 1 hour at room temperature. Proteins were labeled with iTRAQ tags as follows: good prognosis group 114 isobaric tag, and locoregional recurrence group 115 isobaric tag. Then, the iTRAQ-labeled samples were mixed, desalted with Sep-Pak Vac C18 cartridges (Waters, Milford, MA, USA) and dried in a vacuum concentrator.

\section{SCX-RPLC-MS/MS analysis}

The iTRAQ labeling mixed peptides were separated by SCX chromatography using a Polysulfoethyl column $(2.1 \times 100 \mathrm{~mm}, 5 \mu \mathrm{m}, 200 \AA$, The Nest Group, Southborough, MA, USA) with high performance liquid chromatography (HPLC)-20AD system (Shimadzu, Kyoto, Japan). The peptide mixture was added to Buffer A (10 mM KH2PO4 in $25 \%$ acetonitrile, $\mathrm{pH}=2.8$ ), and loaded onto the Polysulfoethyl column. Then, the peptides were separated at a flow rate of $200 \mathrm{~mL} / \mathrm{min}$ for $60 \mathrm{~min}$ with a gradient of $0-80 \%$ Buffer B (Buffer A containing $350 \mathrm{mM} \mathrm{KCl}$, $\mathrm{pH}=2.6$ ). Next, a total of 20 SCX fractions were collected per iTRAQ set and the absorbance at 214 and $280 \mathrm{~nm}$ was monitored. Eventually, the fractions were concentrated by vacuum centrifugation and dissolved in $50 \mu \mathrm{L}$ reversedphase Buffer C (5\% ACN, 0.1\% formic acid), loaded across the ZORBAX 300SB-C18 reversed-phase column (Sigma, $5 \mu \mathrm{m}, 300 \AA, 0.1 \times 150 \mathrm{~mm}$ ) and analyzed on QSTAR XL system (Applied Biosystems) interfaced with HPLC-20AD system. The flow rate of elution was $300 \mathrm{~nL} / \mathrm{min}$ with gradient $5-35 \%$ Buffer D (95\% ACN, 0.1\% formic acid) for $90 \mathrm{~min}$. Survey scans were acquired from $400-1,800 \mathrm{~m} / \mathrm{z}$ for MS with up to 4 precursors selected for MS/MS from $100-2,000 \mathrm{~m} / \mathrm{z}$. The flow chart of the experiment is shown in Figure 1.

\section{Protein identification and relative quantification}

The mass spectra were extracted by ProteinPilot software (version 3.0, Revision 114732, Applied Biosystems) and retrieved from the International Protein Index (IPI) database (version 3.45, Human). Using trypsin as enzyme and methyl methanethiosulfonate of cysteines residues as fixed modification, Paragon algorithm (Applied Biosystems) and Pro Group algorithm (Applied Biosystems) were applied to determine the final identified proteins. Other parameters such as fragment ion mass accuracy, parent ion accuracy, tryptic cleavage specificity, and allowance for number of missed cleavages were provided and processed by ProteinPilot software. Unused ProtScore $>1.3$ as threshold with at least 1 peptide with $95 \%$ confidence was considered as protein identification. Fold changes of $>1.5$ or $<0.66$ between 2 groups were set as the cut-off ratio to designate DEPs. The false discovery rate (FDR) for protein detection was calculated as FDR $=(2 \times$ reverse $) /($ forward + reverse $)$.

\section{Bioinformatics analyses of DEPs}

Identified proteins were mapped to their corresponding UniProt accession numbers using the UniProt webbased portal (www.uniprot.org/mapping). The subcellular location, biological process, and molecular function of the identified proteins were elucidated by the Gene Ontology (GO) analysis using the software of Molecule Annotation System database (MAS 3.0, http://bioinfo.capitalbio.com/ mas3/). ingenuity pathway analysis software (IPA) was used to obtain further insight into potential cellular pathways.

\section{Survival analyses}

We used the Kaplan-Meier (KM) Plotter Online Tool (http://www.kmplot.com) to evaluate the relationship 


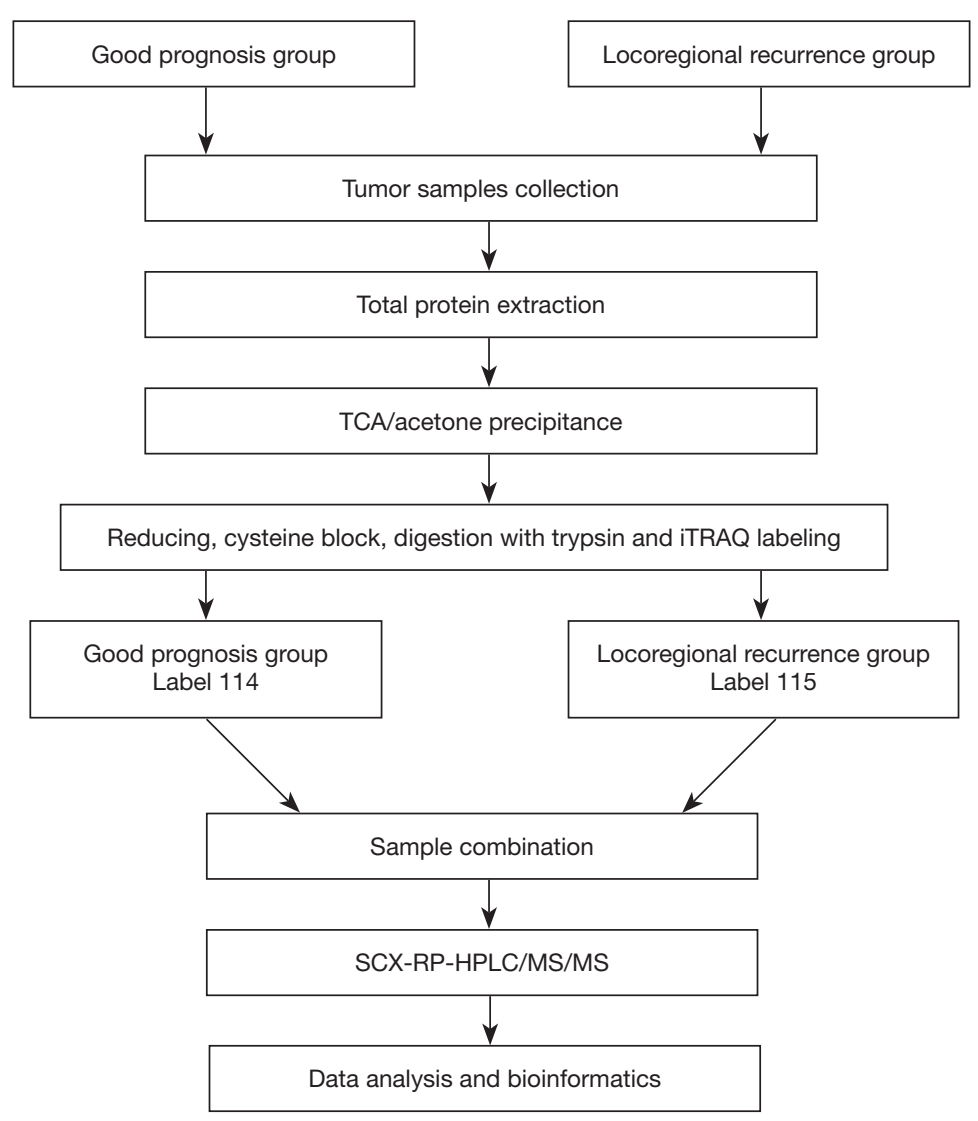

Figure 1 The work flow sheet. iTRAQ, Isobaric tags for relative and absolute quantification; SCX, strong cation exchange; RPLC-MS/MS: reversed phase liquid chromatography-tandem mass spectrometry.

between the differential expression of proteins and survival in patients with ESCC (18). This is a public database containing information from 81 cases of ESCC, allowing the investigation of the relationship between the genes and OS and recurrence-free survival (RFS).

\section{Statistical analysis}

The software SPSS version 13.0 (SPSS Inc., Chicago, IL, USA) was used for statistical analysis. Descriptive statistics [mean \pm standard deviation (SD) for continuous variables and proportion for categorical variables] were used to summarize demographic clinical and pathological characteristics. Numerical variables were compared with unpaired t-test. The nonparametric data of 2 groups were compared using Mann-Whitney $\mathrm{U}$ test. A $\mathrm{P}$ value $<0.05$ was considered statistically significant based on a 2 -sided significance level.

\section{Results}

\section{Clinicopathological features of the fifteen ESCC cases examined}

The overall clinicopathological features of 2 groups are summarized in Table 1. There were no significant differences in age, gender, histological differentiation, tumor location, tumor size, depth of invasion, lymph node status, and vascular invasion between the 2 groups.

\section{Quantitative analysis of LC-MS/MS analysis}

According to the parameter described above, a total of 10,717 distinct peptides corresponding to 449 unique proteins were identified. Global FDR (unused Protscore threshold 1.3) of the combined data was $0.6 \%$ (Table 2). A total of 82 DEPs were found in the locoregional 
Table 1 Clinicopathological features of the 15 ESCC cases examined

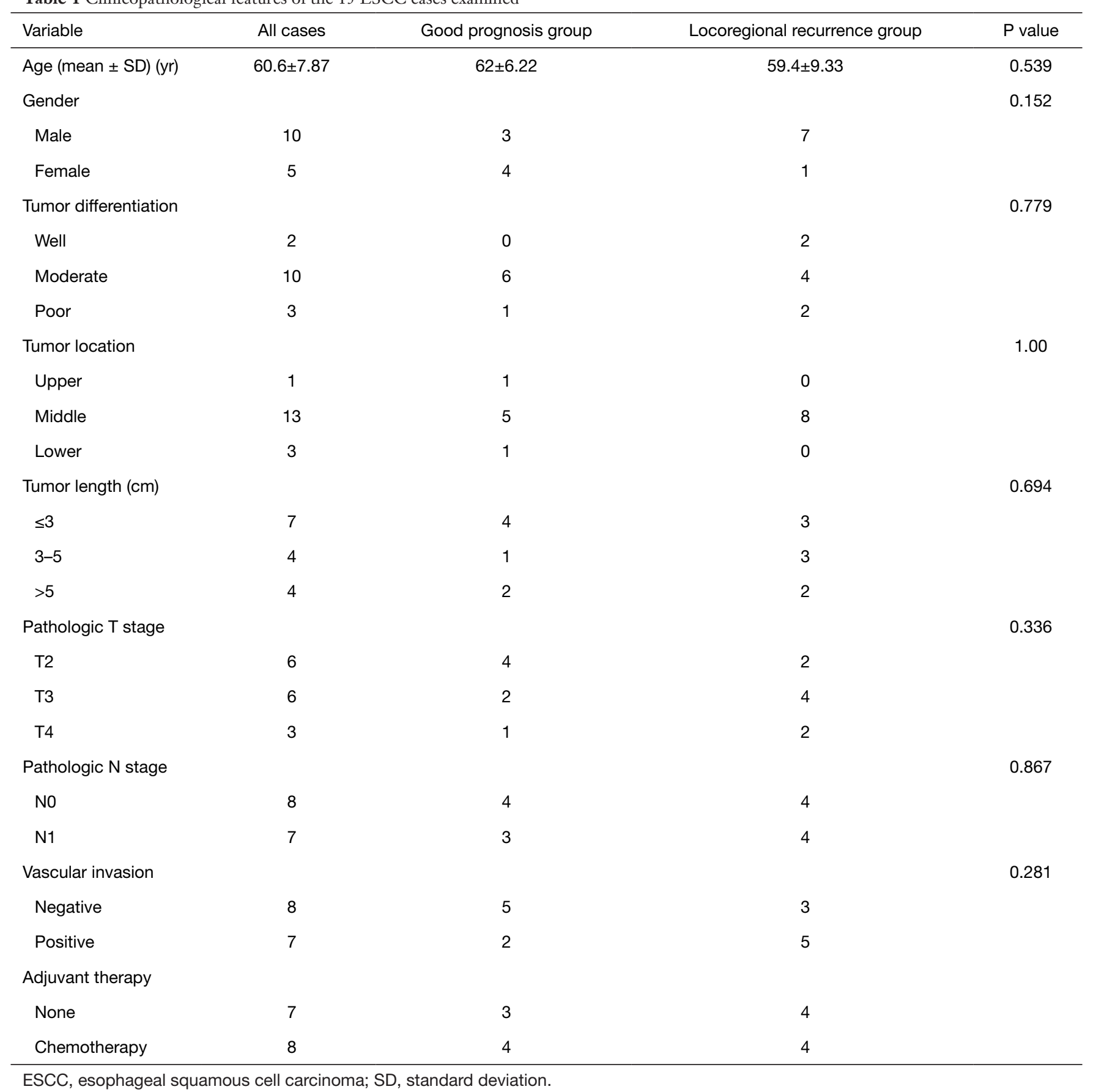

recurrence group as compared with the good prognosis group based on the ITRAQ ratios of peptides identified for these proteins. Among the 82 DEPs, 27 were upregulated $>1.5$-fold and 55 proteins down-regulated $<0.66$-fold (Table 3).

\section{GO annotation and protein classification}

We uploaded all 82 DEPs to the online software MAS to identify GO categories based on subcellular localization, biological process, and molecular function. In the analysis 
Table 2 Summary statistics of proteomic results of IPI data

\begin{tabular}{lccccc}
\hline $\begin{array}{l}\text { Unused [Conf] } \\
\text { cutoff }\end{array}$ & Proteins detected Proteins before grouping & Distinct peptides & Spectra identified & \% Total spectra & Global FDR \\
\hline$>2.0[99]$ & 296 & 476 & 8,876 & 16,217 & 21.1 \\
$>1.3[95]^{*}$ & 449 & 673 & 10,717 & 18,843 & 24.6 \\
$>0.47[66]$ & 549 & 949 & 12,016 & 20,560 & 26.8 \\
\hline
\end{tabular}

*This row shows the unused Protscore threshold applied in this paper and the corresponding statistical result. FDR, false discovery rate; IPI, international protein index.

Table 3 DEPs identified by iTRAQ-based quantitative proteomics

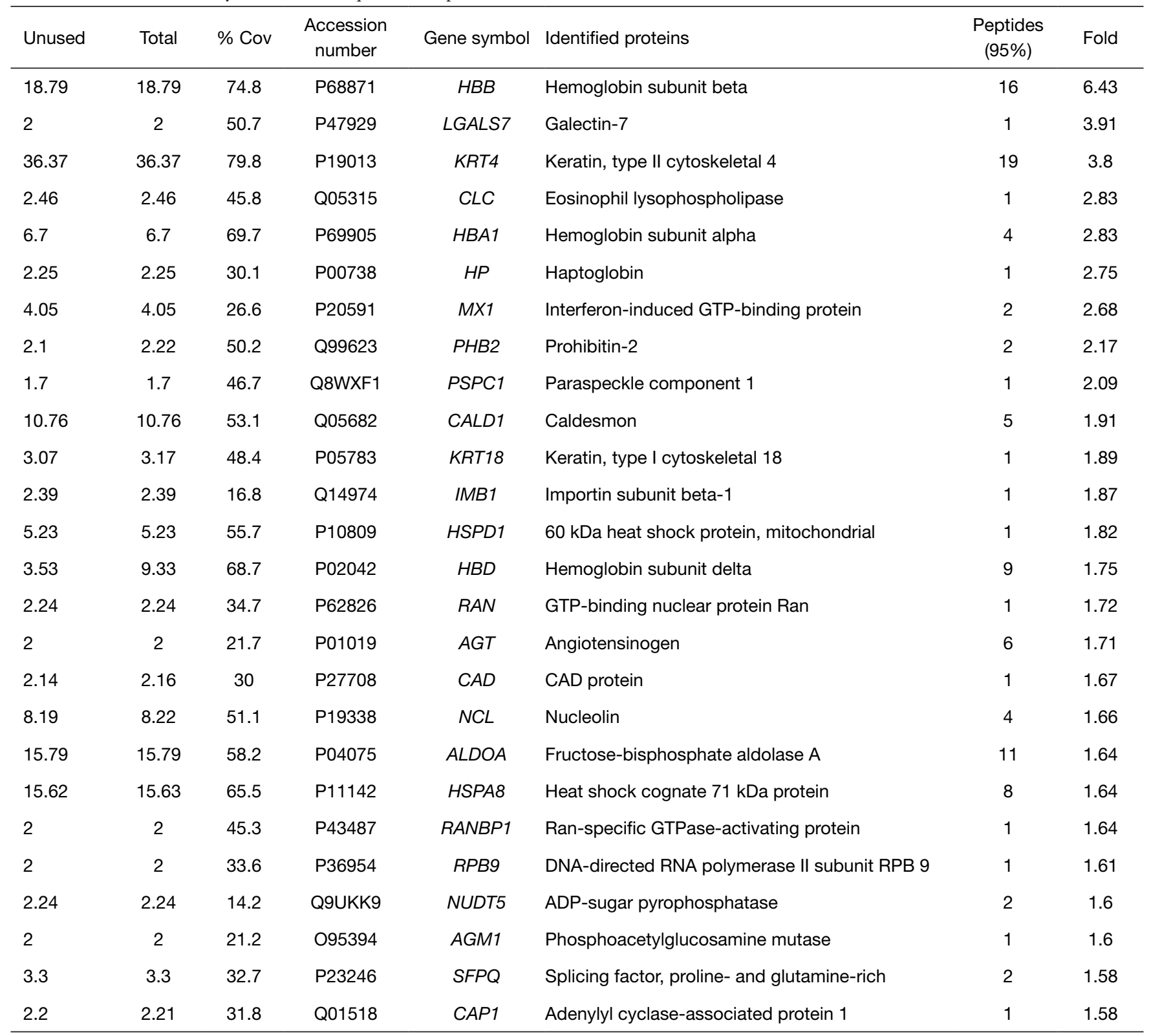

Table 3 (continued) 
Table 3 (continued)

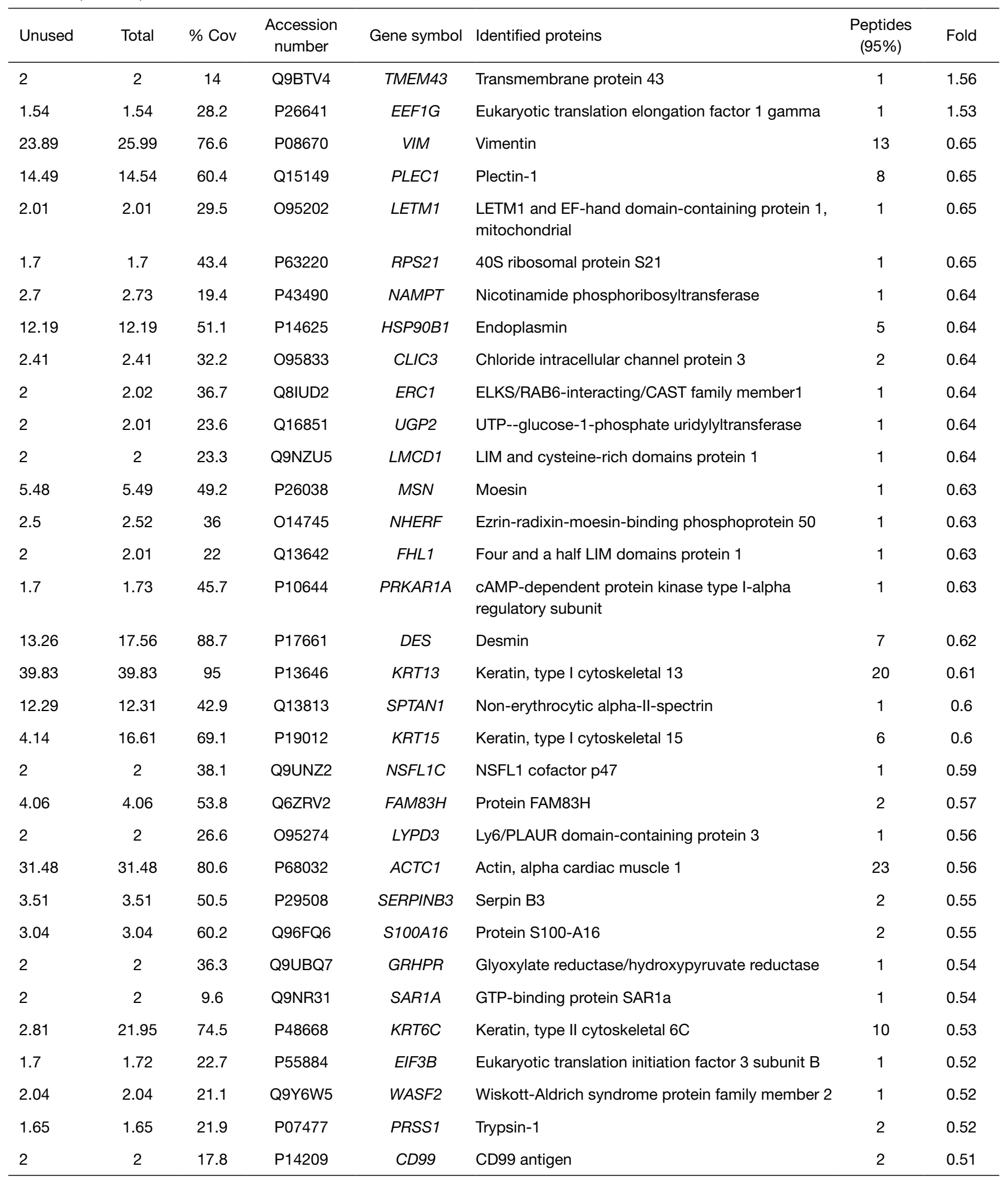

Table 3 (continued) 
Table 3 (continued)

\begin{tabular}{|c|c|c|c|c|c|c|c|}
\hline Unused & Total & $\% \mathrm{Cov}$ & $\begin{array}{c}\text { Accession } \\
\text { number }\end{array}$ & Gene symbol & Identified proteins & $\begin{array}{c}\text { Peptides } \\
(95 \%)\end{array}$ & Fold \\
\hline 2.49 & 2.5 & 25.3 & Q16787 & LAMA3 & Laminin subunit alpha-3 & 1 & 0.51 \\
\hline 9.46 & 9.46 & 57.8 & Q9UBG3 & CRNN & Cornulin & 5 & 0.5 \\
\hline 4.59 & 4.59 & 45.2 & Q06830 & PRDX1 & Peroxiredoxin-1 & 2 & 0.48 \\
\hline 5.26 & 5.26 & 31.7 & P51884 & LUM & Lumican & 2 & 0.47 \\
\hline 2.62 & 2.62 & 73.8 & P27482 & CALML3 & Calmodulin-like protein 3 & 1 & 0.47 \\
\hline 6.61 & 6.61 & 35.7 & P24821 & TNC & Tenascin C & 3 & 0.41 \\
\hline 11.64 & 25.15 & 81.8 & P08779 & KRT16 & Keratin, type I cytoskeletal 16 & 9 & 0.4 \\
\hline 22.28 & 26.3 & 74.2 & P13647 & KRT5 & Keratin, type II cytoskeletal 5 & 13 & 0.37 \\
\hline 14.61 & 14.61 & 83.1 & Q01995 & TAGLN & Transgelin & 6 & 0.32 \\
\hline 7.02 & 7.04 & 29.2 & Q00610 & CLTC & Clathrin heavy chain 1 & 3 & 0.3 \\
\hline 3.24 & 3.24 & 62.9 & P60660 & MYL6 & Myosin light polypeptide 6 & 2 & 0.28 \\
\hline 5.12 & 5.13 & 44.1 & P12110 & COL6A2 & Collagen alpha-2(VI) chain & 4 & 0.28 \\
\hline
\end{tabular}

DEPs, differentially expressed proteins; iTRAQ, Isobaric tags for relative and absolute quantification.

of biological process, most up-regulated DEPs mainly play roles in interspecies interaction between organisms, signal transduction, and oxygen transport. The downregulated DEPs were significantly enriched in epidermis development, cell motility, muscle development, and cell-cell signaling (Table 4). For molecular function, the up-regulated DEPs were enriched in protein binding, nucleotide binding, and metal ion binding, and the down-regulated DEPs were enriched in protein binding, structural constituent of cytoskeleton, and calcium ion binding (Table 4). In addition, GO cell component analysis displayed that the up-regulated DEPs were significantly enriched in cytoplasm, nucleus, cytosol, hemoglobin complex, and nuclear matrix. The down-regulated DEPs enriched in cytoplasm, plasma membrane, nucleus, cytoskeleton, and extracellular region (Table 4).

In Ingenuity software, 82 DEPs were further analyzed to identify biological functions, significant networks, and canonical pathways. The IPA analysis identified "cancer" as one of the main diseases associated with the DEPs by the biological functions. Moreover, multiple categories of biological functions, such as cell movement, cell assembly and tissue, cell function and maintenance, cell growth and proliferation, tissue development, cell death and 
Table 4 GO analysis of the 82 DEPs (top 5 shown)

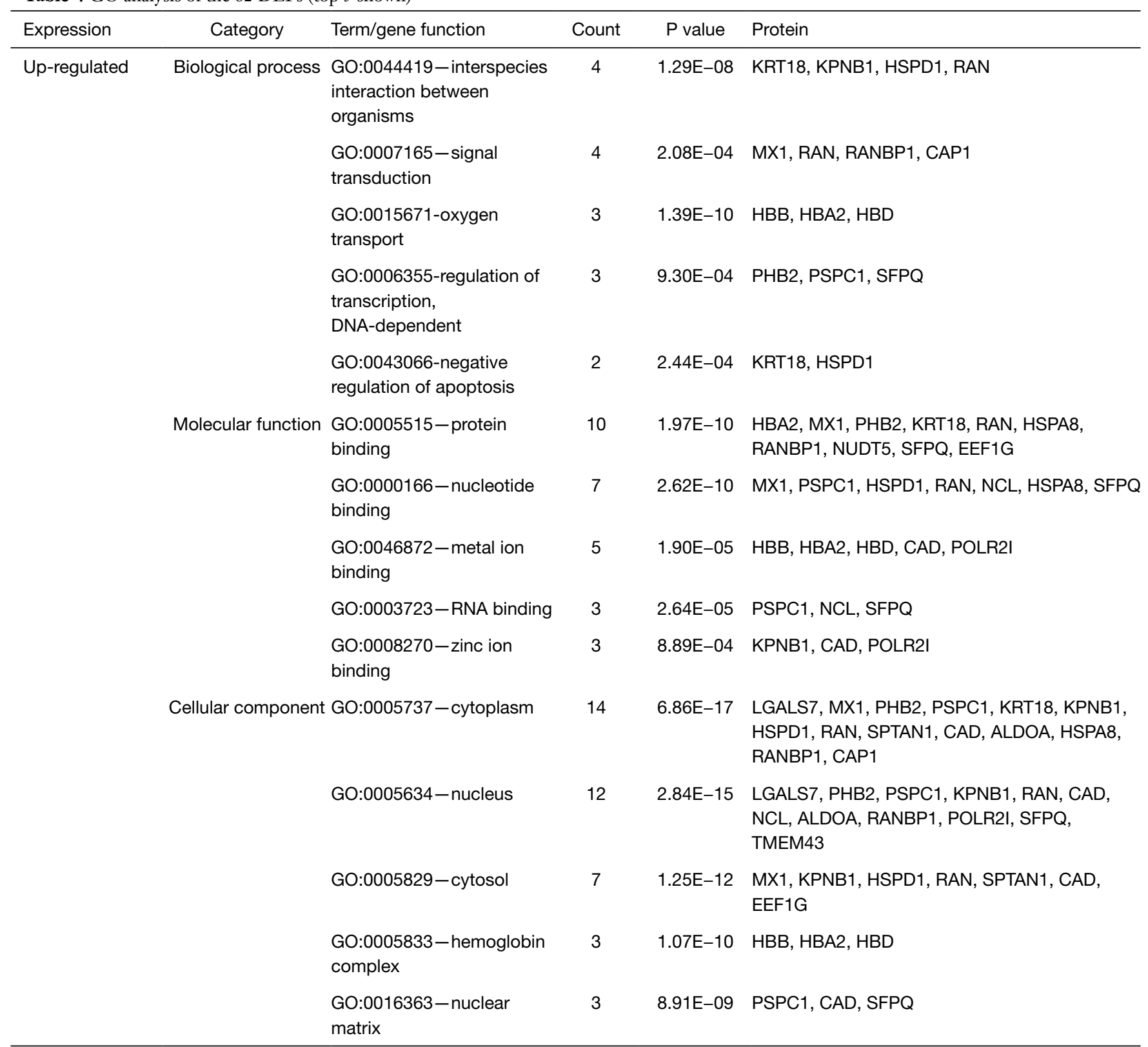

Table 4 (continued) 
Table 4 (continued)

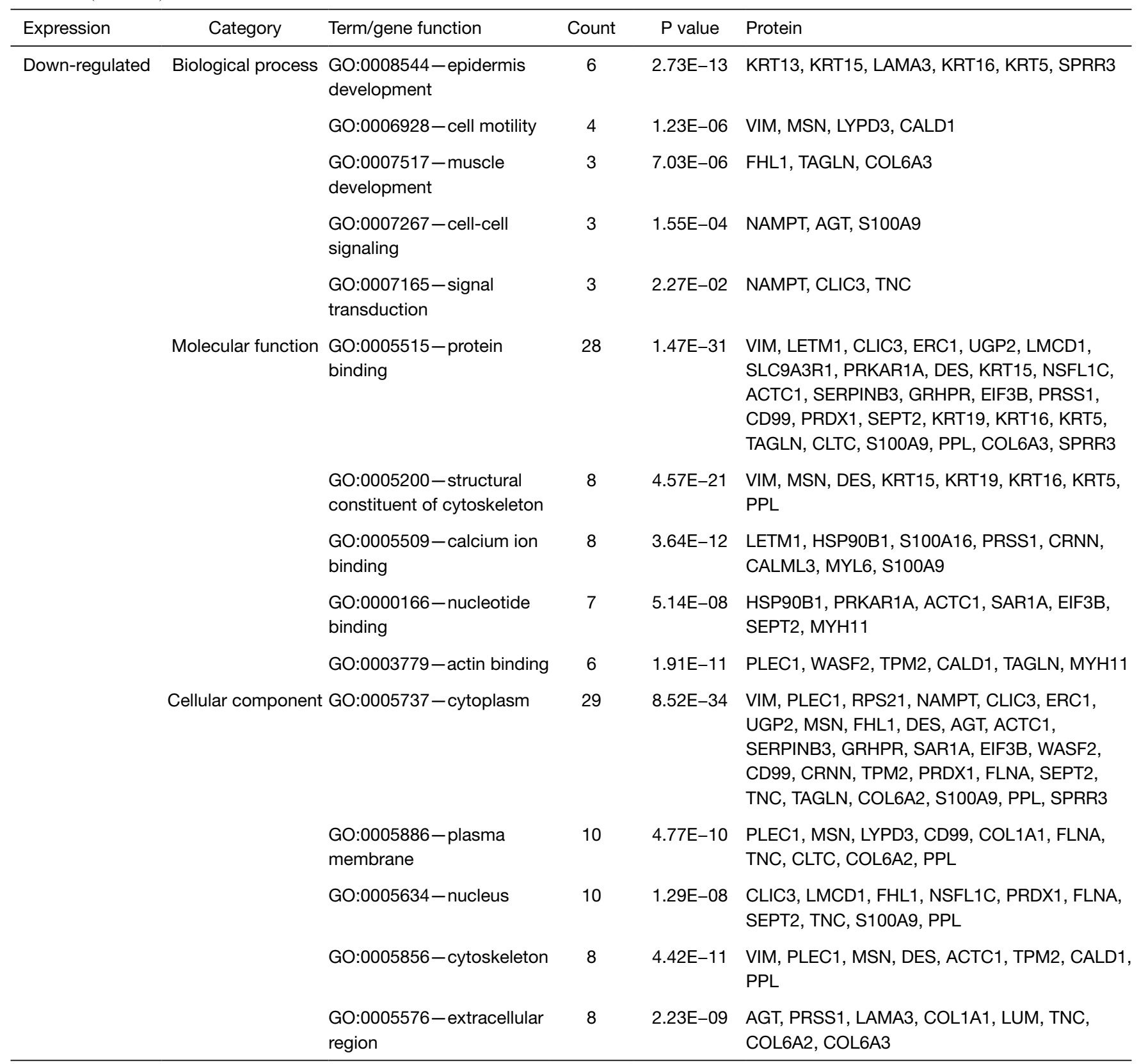

GO, Gene Ontology; DEPs, differentially expressed proteins.

survival, carbohydrate metabolism, DNA replication, and recombination and repair, are related to cancer development. The biological functions are shown in Figure $2 A$. Furthermore, IPA interaction analysis detected 35 significant canonical pathways including RNA signaling, calcium signaling, ILK signaling, actin cytoskeleton signaling, and RhoA signaling among other pathways
(Figure 2B). In addition, IPA interaction analysis found 3 significant pathway networks (Table 5 and Figure 3). These significant networks functioned in dermatological diseases and conditions, hereditary disorder, connective tissue disorders, cell death and survival, cancer, cellular function and maintenance, and cellular movement (Table 5). The key molecules in each network are listed in Figure 3. The 

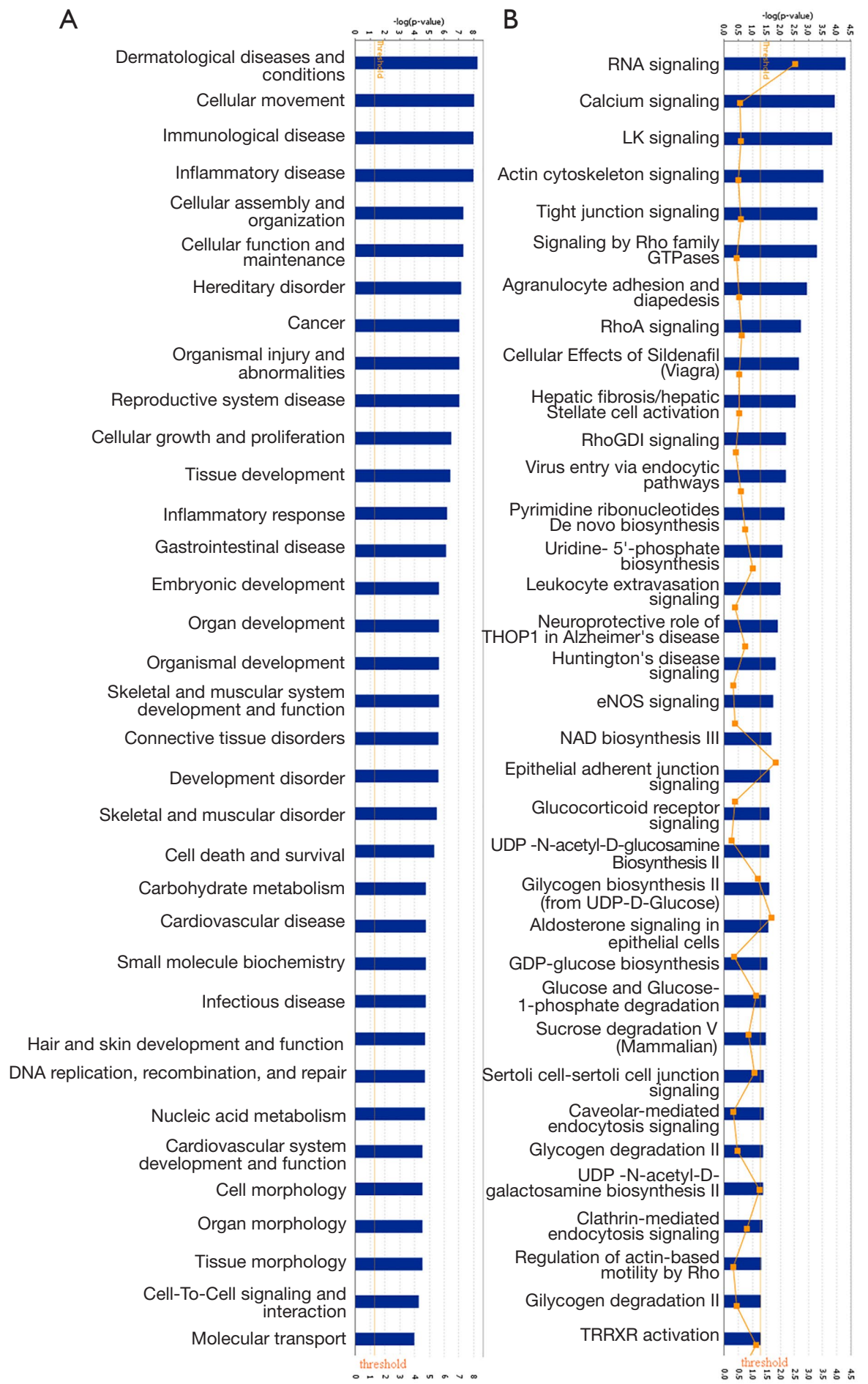

Figure 2 Biofunctions and pathway analysis of the DEPs using IPA. (A) Biofunctions in which the DEPs are involved. (B) Canonical pathways that involved the DEPs. DEPs, differentially expressed proteins; IPA, ingenuity pathway analysis software. 
Table 5 The statistically significant pathway networks that involved the 82 DEPs

\begin{tabular}{|c|c|c|c|}
\hline Networks & Score & $\begin{array}{c}\text { Focused } \\
\text { molecules }\end{array}$ & DEPs in network \\
\hline $\begin{array}{l}\text { Dermatological diseases and conditions, } \\
\text { hereditary disorder, connective tissue disorders }\end{array}$ & 51 & 23 & $\begin{array}{l}\text { CALD1, CAP1, COL6A2, DES, FLNA, KRT4, KRT5, KRT13, KRT15, } \\
\text { KRT16, KRT18, KRT19, LAMA3, MSN, MYH11, MYL6, NAMRT, } \\
\text { PLEC1, PPL, SPTAN1, TPM2, VIM, WASF2 }\end{array}$ \\
\hline $\begin{array}{l}\text { Cell death and survival, cancer, cellular } \\
\text { function and maintenance }\end{array}$ & 38 & 18 & $\begin{array}{l}\text { ACTC1, CD99, CLTC, ERC1, HBB, HBD, HSP90B1, HSPA8, } \\
\text { HSPD1, KPNB1, LGSLS7, NCL, NSFL1C, PRDX1, PSPC1, RAN, } \\
\text { RPS21, SFPQ }\end{array}$ \\
\hline
\end{tabular}

DEPs, differentially expressed proteins.

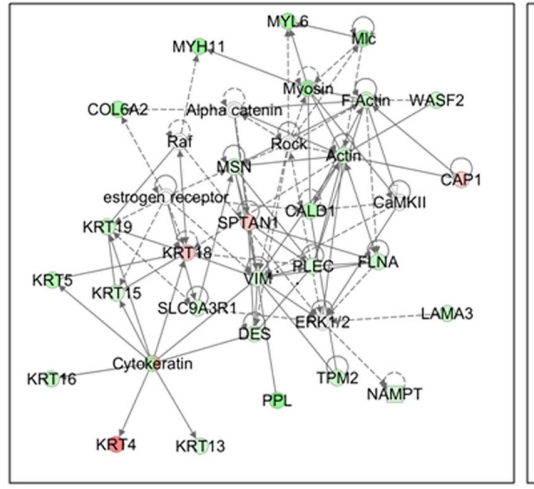

Network1

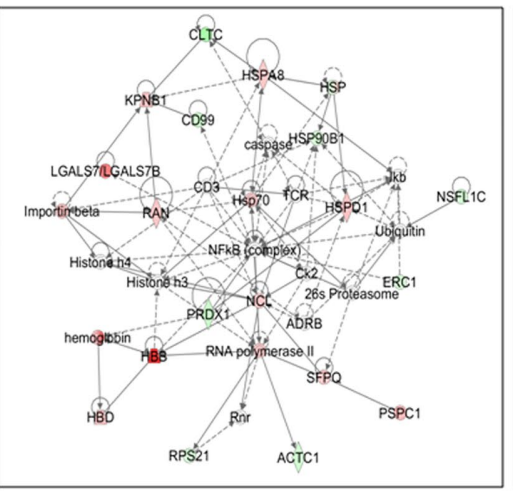

Network2

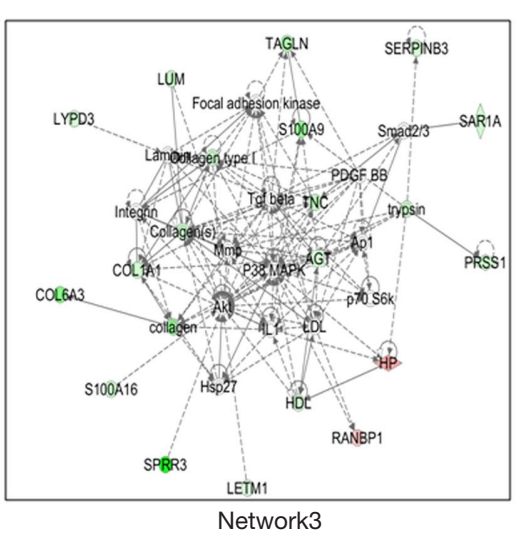

Relationship and shapes: direct interaction (-), Indirect interaction ( ----), Inhibits ( $\longrightarrow$ ), Acts on ( $\longrightarrow$ ), Complex/Group ( (O) ),

Peptidase $(\diamond)$, Enzyme $(\diamond)$, Cytokine $(\square)$, Transporter $(\square)$, Transcription regulator ( $\bigcirc)$, Translation regulator $(\square)$,

Trans-membrane receptor $(\mathrm{O})$, Other $(\mathrm{O})$.

Figure 3 The 3 significant pathway networks that involved the 82 DEPs. The red node represents up-regulated protein and the green node represents down-regulated protein. Uncolored nodes were not identified as DEPs, but were integrated into the computationally generated networks on the basis of the evidence stored in the IPA knowledge memory indicating a relevance to this network. DEPs, differentially expressed proteins; IPA, ingenuity pathway analysis software.

proteins CALD1, CAP1, DES, FLNA, KRT18, PLEC1, nonerythroid spectrin II (SPTAN1), TPM2, and VIM play key roles in the network 1. The proteins HBB, HSPA8, HSPD1, KPNB1, LGSLS7, NCL, PRDX1, and RAN play key roles in network 2 . The proteins angiotensinogen (AGT), COL1A1, S100A9 and TAGLN play important roles in network 3 .

\section{The association of differential expression of proteins and patient survival}

The key proteins were validated using ESCC data in the
KM Plotter Online Tool. Among them, SPTAN1 was positively associated with the RFS (Figure $4 A$ ), and high expression of SPTAN1 tended to have favorable OS (Figure 4B). The AGT protein was found to be negatively associated with RFS and high expression of AGT tended to have poor OS (Figure 4C,D). Other proteins were not found to be associated with OS and RFS.

\section{Discussion}

The outcomes of patients with ESCC treated primarily with surgery have demonstrated heterogeneity. Postoperative 
A

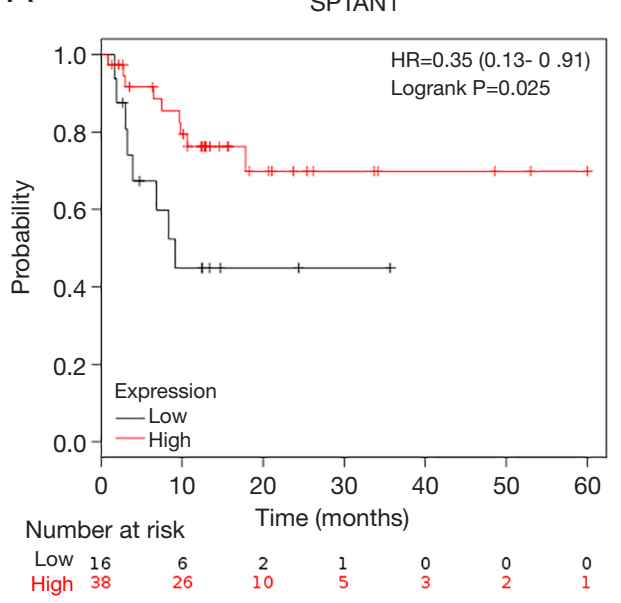

C

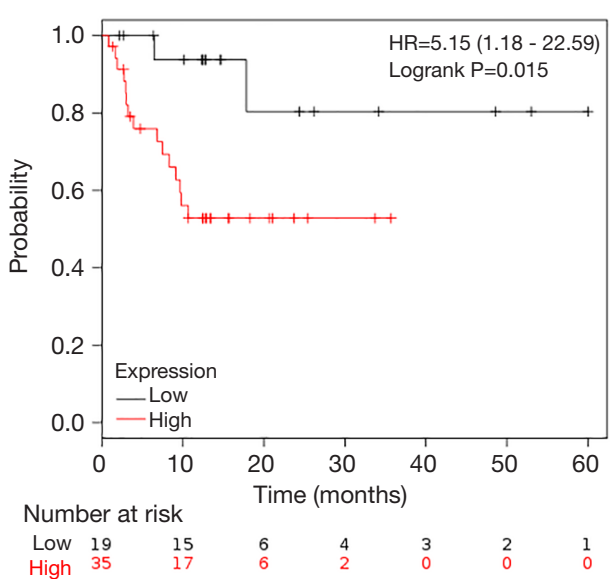

B

SPTAN1
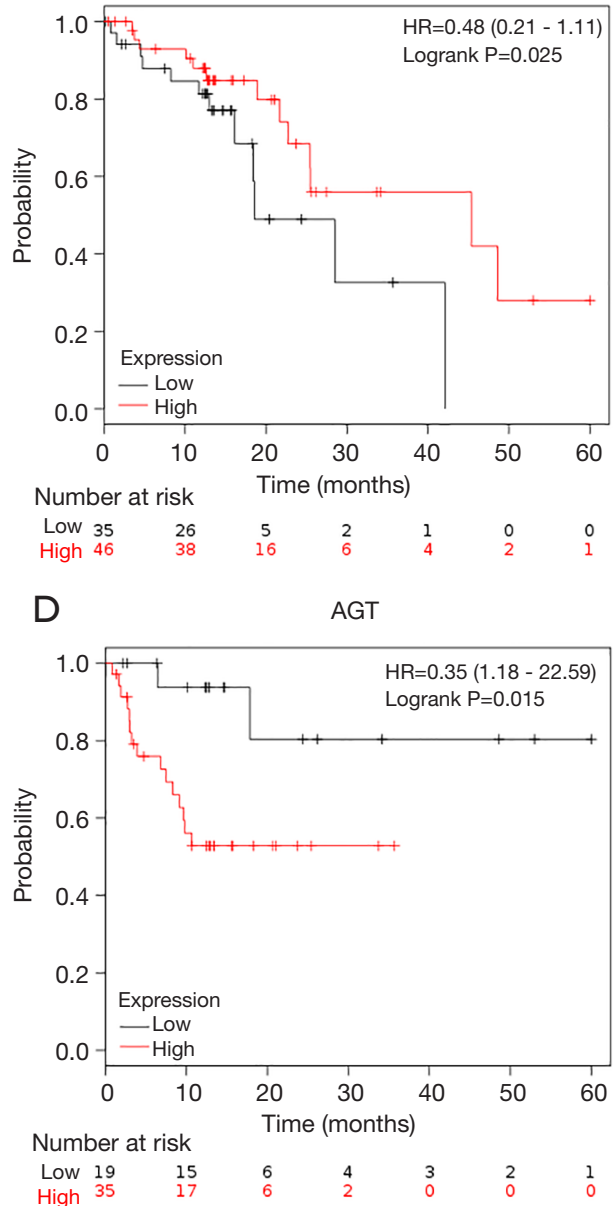

Figure $4 \mathrm{KM}$ survival analysis for the relationship between survival and proteins expression of SPTAN1 and AGT in ESCC was performed using ESCC data of KM Plotter Online Tool. (A) Association of SPTAN1 with RFS in ESCC, (B) association of SPTAN1 with OS in ESCC, (C) association of AGT with RFS in ESCC, (D) association of AGT with OS in ESCC. KM, Kaplan-Meier; ESCC, esophageal squamous cell carcinoma; RFS, recurrence-free survival; OS, overall survival.

radiotherapy (PORT) combined with chemotherapy is one of the important strategies that decreases locoregional recurrence and then increases OS in patients with ESCC. If we could predict the high risk of locoregional recurrence, then perhaps we could correctly identify patients who would benefit from PORT and avoid radiation-associated morbidity in the patients who could be cured with surgery alone. In current clinical practice, we mostly rely on clinicopathological characteristics, such as pathological tumour-node-metastasis (pTNM) staging, especially number of metastatic lymph nodes, to stratify high-risk subgroups of distant metastasis and local recurrent patients for improving treatment after surgery (19). Some studies have investigated expression of proteins in certain signaling pathways by immunohistochemical to predict poor survival (20).

Recently, advances in proteomic and genomic technologies have enabled us to identify differentially expressed proteins and genes and further explore the potential biomarkers and therapeutic targets for cancers $(14,21)$. Comparative proteomic analysis of human ESCC with poor and good prognosis is one of the most direct and convincing ways to find biomarkers for its prognosis. In this analysis, we focused on the prediction of locoregional recurrence in ESCC patients after radical operation. We used the quantitative proteomic technique iTRAQ to analyze tumor tissue from a locoregional recurrence 
group and good prognosis group of postoperative participants with ESCC for the discovery of proteins with potential as candidates for locoregional recurrencerelated biomarkers. The current study identified a total of 82 DEPs between the locoregional recurrence group and good prognosis group, and revealed multiple categories of biological functions related to tumor recurrence, including cellular movement, cellular function and maintenance, cellular assembly and organization, cellular growth and proliferation, tissue development, cell death and survival, carbohydrate metabolism, and DNA replication recombination and repair.

Using IPA interaction analysis, we found 3 significant networks involving 57 DEPs. Of these, 24 proteins had not been previously investigated in esophageal carcinoma, including CALD1, COL6A2, MYH11, MYL6, SPTAN1, WASF2, ACTC1, CLTC, ERC1, HBD, HSP90B1, HSPD1, NSFL1C, PSPC1, SFPQ, RPS21, AGT, COL6A3, LETM1, PRSS1, RANBP1, S100A16, SAR1A, and SERPINB3. In addition, we identified 2 proteins that were associated with RFS in ESCC. We found that SPTAN1 was positively associated with RFS and AGT was negatively associated with RFS; expression of SPTAN1 tended to have favorable OS while AGT tended to have poor OS.

The SPTAN1 protein is an important cytoskeletal protein that ensures important cellular properties, including polarity and cell stabilization. It is also involved in cell adhesion, cell-to-cell contact, and apoptosis $(22,23)$. Expression changes of SPTAN1 have been found in a variety of tumors, and its expression appears to have opposite effects in different tumors. On the one hand, most data suggest over-expression of SPTAN1 in cancer and progression; on the other hand, reduced expression of SPTAN1 has also been observed in tumors (24). Ackermann et al. (25) investigated expression of SPTAN1 in 189 patients with surgically resected colorectal tumors (CRCs) using immunohistochemistry. They found that SPTAN1 expression was higher in early stage CRCs and decreased gradually in advanced stages. In addition, the expression of SPTAN1 in metastatic CRCs was lower than that in nonmetastatic CRCs. Knockdown of SPTAN1 in CRC cell lines demonstrated weakened cell-to-cell contact, which may indicate that tumor cells expressing low levels of SPTAN1 detach from their primary tumor sites and metastasize more easily (25). Bii et al. (26) also found SPTAN1 downregulation in lung metastasis tissue of patient with prostate cancer. Our study found that down-regulation of SPTAN1 was related to progression in ESCC. However, further analysis of SPTAN1 is required to confirm its potential value as a predictive marker of cancer invasiveness and as a candidate protein for targeted therapy.

The precursor of angiotensin peptide, AGT, is the only known naturally occurring rennin substrate (27). Recent studies have implied that AGT is associated with inflammation and in vitro inhibition of human endothelial cell proliferation, cell migration, and angiogenesis (28). Zhang et al. (29) downloaded the original RNA-Seq, clinical information, and Illumina Human Methylation 27 Chip data associated with gastric cancer (GC) from The Cancer Genome Atlas (TCGA) database using the gdcclient tool, and screened the differentially expressed genes. They found AGT was up-regulated and the expression of AGT was associated with the prognosis of GC patients. Sugimoto et al. found AGT gene polymorphism increased GC risk, especially intestinal GC, indicating that the renin-angiotensin (RA) system plays an important role in the pathogenesis of GC (30). With a stringent cut-off of 1.5 -fold up-regulation in the current study, we found that AGT protein up-regulated in the locoregional recurrence group compared to the good prognosis group. To the best of our knowledge, this was the first study to report that high expression of AGT and low expression of SPTAN1 were associated with relapse in ESCC after radical esophagectomy. Further studies are currently underway to validate the exact predictive value of expression of AGT and SPTAN1 in ESCC and to explore the functions of these proteins.

In this study, we used proteomics to identify DEPs and hope to find potential biomarkers for predicting prognosis of ESCC. Up to date, proteomics have been widely used to investigate therapeutic efficacy and mechanism of action of drugs. Li et al. (31) applied quantitative proteomics to investigate the anti-tumor activity of levodopa on ESCC to the molecular mechanisms. Using comparative proteomic to screen DEPs between KYSE150 cells treated with $600 \mu \mathrm{M}$ levodopa and untreated KYSE150 cells, they found levodopa could down regulate oxidative phosphorylation, non-alcoholic fatty liver disease, and Parkinson's disease pathways. MA et al. (32) used iTRAQ technique to screen serum differentially expressed proteins associated with chemoradiotherapeutic efficacy. Proteomics results revealed that prior to chemoradiotherapy, the expression level of integrin-linked kinase was significantly upregulated in patients with ESCC, compared with that of the control group, and significantly downregulated in the chemoradiotherapy-sensitive group, compared with the 
chemoradiotherapy-resistant group.

Our work had some limitations, this was a computer simulation analysis using data from several sources, so it was mandatory to evaluate these findings in a homogenous data set. Furthermore, these results should be considered as hypotheses generating and confirmation is required through independent datasets and in prospective and retrospective studies.

\section{Conclusions}

Our results demonstrated quantitative proteomics as an effective discovery tool to identify biomarkers for prognosis prediction in ESCC. There were 2 proteins identified in this study that may be potential biomarkers for prediction of locoregional recurrence in ESCC after radical esophagectomy. However, more studies with large populations of individuals with ESCC are needed to validate these potential biomarkers.

\section{Acknowledgments}

Funding: This study was supported by the Shanghai Committee of Science and Technology, Natural Science Foundation of Shanghai (15ZR1432100).

\section{Footnote}

Reporting Checklist: The authors have completed the REMARK reporting checklist. Available at https://dx.doi. org/10.21037/jgo-21-278

Data Sharing Statement: Available at https://dx.doi. org/10.21037/jgo-21-278

Conflicts of Interest: All authors have completed the ICMJE uniform disclosure form (available at https://dx.doi. org/10.21037/jgo-21-278). The authors have no conflicts of interest to declare.

Ethical Statement: The authors are accountable for all aspects of the work in ensuring that questions related to the accuracy or integrity of any part of the work are appropriately investigated and resolved. All procedures performed in this study involving human participants were in accordance with the Declaration of Helsinki (as revised in 2013). The study was approved by the appropriate institutional review board of Fudan
University Cancer Center and informed consent was taken from all the patients.

Open Access Statement: This is an Open Access article distributed in accordance with the Creative Commons Attribution-NonCommercial-NoDerivs 4.0 International License (CC BY-NC-ND 4.0), which permits the noncommercial replication and distribution of the article with the strict proviso that no changes or edits are made and the original work is properly cited (including links to both the formal publication through the relevant DOI and the license). See: https://creativecommons.org/licenses/by-nc-nd/4.0/.

\section{References}

1. Nakajima M, Kato H. Treatment options for esophageal squamous cell carcinoma. Expert Opin Pharmacother 2013;14:1345-54.

2. Mariette C, Balon JM, Piessen G, et al. Pattern of recurrence following complete resection of esophageal carcinoma and factors predictive of recurrent disease. Cancer 2003;97:1616-23.

3. Yamashita K, Watanabe M, Mine S, et al. Patterns and Outcomes of Recurrent Esophageal Cancer After Curative Esophagectomy. World J Surg 2017;41:2337-44.

4. Macdonald JS, Smalley SR, Benedetti J, et al. Chemoradiotherapy after surgery compared with surgery alone for adenocarcinoma of the stomach or gastroesophageal junction. N Engl J Med 2001;345:725-30.

5. van Hagen P, Hulshof MC, van Lanschot JJ, et al. Preoperative chemoradiotherapy for esophageal or junctional cancer. N Engl J Med 2012;366:2074-84.

6. Kleinberg L, Forastiere AA. Chemoradiation in the management of esophageal cancer. J Clin Oncol 2007;25:4110-7.

7. Tepper J, Krasna MJ, Niedzwiecki D, et al. Phase III trial of trimodality therapy with cisplatin, fluorouracil, radiotherapy, and surgery compared with surgery alone for esophageal cancer: CALGB 9781. J Clin Oncol 2008;26:1086-92.

8. Fareed KR, Kaye P, Soomro IN, et al. Biomarkers of response to therapy in oesophago-gastric cancer. Gut 2009;58:127-43.

9. Lin DC, Du XL, Wang MR. Protein alterations in ESCC and clinical implications: a review. Dis Esophagus 2009;22:9-20.

10. Lee JM, Kohn EC. Proteomics as a guiding tool for more effective personalized therapy. Ann Oncol 2010;21 
Suppl 7:vii205-10.

11. Dunn BK, Wagner PD, Anderson D, et al. Molecular markers for early detection. Semin Oncol 2010;37:224-42.

12. Pan S, Brentnall TA, Kelly K, et al. Tissue proteomics in pancreatic cancer study: discovery, emerging technologies, and challenges. Proteomics 2013;13:710-21.

13. Yang W, Cai Q, Lui VW, et al. Quantitative proteomics analysis reveals molecular networks regulated by epidermal growth factor receptor level in head and neck cancer. J Proteome Res 2010;9:3073-82.

14. Qi YJ, Chao WX, Chiu JF. An overview of esophageal squamous cell carcinoma proteomics. J Proteomics 2012;75:3129-37.

15. Zhang J, Wang K, Zhang J, et al. Using proteomic approach to identify tumor-associated proteins as biomarkers in human esophageal squamous cell carcinoma. J Proteome Res 2011;10:2863-72.

16. Cai XW, Yu WW, Yu W, et al. Tissue-based quantitative proteomics to screen and identify the potential biomarkers for early recurrence/metastasis of esophageal squamous cell carcinoma. Cancer Med 2018;7:2504-17.

17. Bradford MM. A rapid and sensitive method for the quantitation of microgram quantities of protein utilizing the principle of protein-dye binding. Anal Biochem 1976;72:248-54.

18. Györffy B, Lanczky A, Eklund AC, et al. An online survival analysis tool to rapidly assess the effect of 22,277 genes on breast cancer prognosis using microarray data of 1,809 patients. Breast Cancer Res Treat 2010;123:725-31.

19. Wei C, Deng WY, Li N, et al. Lymph Node Ratio as an Alternative to the Number of Metastatic Lymph Nodes for the Prediction of Esophageal Carcinoma Patient Survival. Dig Dis Sci 2015;60:2771-6.

20. Zhu Z, Yu W, Fu X, et al. Phosphorylated AKT1 is associated with poor prognosis in esophageal squamous cell carcinoma. J Exp Clin Cancer Res 2015;34:95.

21. Chen FF, Zhang SR, Peng H, et al. Integrative genomics analysis of hub genes and their relationship with prognosis and signaling pathways in esophageal squamous cell carcinoma. Mol Med Rep 2019;20:3649-60.

Cite this article as: $\mathrm{Yu} W W, \mathrm{Fu} \mathrm{XL}$, Cai XW, Sun MH, Guo YM. Identification of differentially expressed proteins in the locoregional recurrent esophageal squamous cell carcinoma by quantitative proteomics. J Gastrointest Oncol 2021;12(3):9911006. doi: 10.21037/jgo-21-278
22. Machnicka B, Czogalla A, Hryniewicz-Jankowska A, et al. Spectrins: a structural platform for stabilization and activation of membrane channels, receptors and transporters. Biochim Biophys Acta 2014;1838:620-34.

23. Bennett V, Baines AJ. Spectrin and ankyrin-based pathways: metazoan inventions for integrating cells into tissues. Physiol Rev 2001;81:1353-92.

24. Ackermann A, Brieger A. The Role of Nonerythroid Spectrin $\alpha$ II in Cancer. J Oncol 2019;2019:7079604.

25. Ackermann A, Schrecker C, Bon D, et al. Downregulation of SPTAN1 is related to MLH1 deficiency and metastasis in colorectal cancer. PLoS One 2019;14:e0213411.

26. Bii VM, Collins CP, Hocum JD, et al. Replicationincompetent gammaretroviral and lentiviral vector-based insertional mutagenesis screens identify prostate cancer progression genes. Oncotarget 2018;9:15451-63.

27. Lynch KR, Peach MJ. Molecular biology of angiotensinogen. Hypertension 1991;17:263-9.

28. Bouquet C, Frau E, Opolon P, et al. Systemic administration of a recombinant adenovirus encoding a HSA-Angiostatin kringle 1-3 conjugate inhibits MDA-MB-231 tumor growth and metastasis in a transgenic model of spontaneous eye cancer. Mol Ther 2003; 7:174-84.

29. Zhang C, Liang Y, Ma MH, et al. KRT15, INHBA, MATN3, and AGT are aberrantly methylated and differentially expressed in gastric cancer and associated with prognosis. Pathol Res Pract 2019;215:893-9.

30. Sugimoto M, Furuta T, Shirai N, et al. Role of angiotensinogen gene polymorphism on Helicobacter pylori infection-related gastric cancer risk in Japanese. Carcinogenesis 2007;28:2036-40.

31. Li Z, Li X, He X, et al. Proteomics Reveal the Inhibitory Mechanism of Levodopa Against Esophageal Squamous Cell Carcinoma. Front Pharmacol 2020;11:568459.

32. Ma XL, Yao H, Wang X, et al. ILK predicts the efficacy of chemoradiotherapy and the prognosis of patients with esophageal squamous cell carcinoma. Oncol Lett 2019;18:4114-25.

(English Language Editor: J. Jones) 